\title{
The Epidemiology of Irritable Bowel Syndrome in North America: A Systematic Review
}

\author{
Yuri A. Saito, M.D., M.P.H., Philip Schoenfeld, M.S.Ed., M.Sc. (Epi), and G. Richard Locke, III, M.D. \\ Division of Gastroenterology and Hepatology, Mayo Clinic, Rochester, Minnesota; and Division of \\ Gastroenterology, University of Michigan School of Medicine and VA Center for Excellence in Health \\ Services Research, Ann Arbor, Michigan
}

OBJECTIVE: The aim of this study was to systematically review published literature about the prevalence, incidence, and natural history of irritable bowel syndrome (IBS) in North America.

METHODS: A computer-assisted search of MEDLINE, EMBASE, and Current Contents/Science Edition databases was performed independently by two investigators. Study selection criteria included: 1) North American populationbased sample of adults; 2) objective diagnostic criteria for IBS (i.e., Rome or Manning criteria); and 3) publication in full manuscript form in English. Eligible articles were reviewed in a duplicate and independent manner. Data extracted were converted into individual tables and presented in descriptive form.

RESULTS: The prevalence of IBS in North America ranges from $3 \%$ to $20 \%$, with most prevalence estimates ranging from $10 \%$ to $15 \%$. The prevalences of diarrhea-predominant and constipation-predominant IBS are both approximately $5 \%$. Published prevalence estimates by gender range from $2: 1$ female predominance to a ratio of $1: 1$. Constipationpredominant IBS is more common in female individuals. The prevalence of IBS varies minimally with age. No true population-based incidence studies or natural history studies were found. In one cohort surveyed on two occasions $1 \mathrm{yr}$ apart, 9\% of subjects who were free of IBS at baseline reported IBS at follow-up producing an onset rate of 67 per 1000 person-years. In all, $38 \%$ of patients meeting criteria for IBS did not meet IBS criteria at 1-yr follow-up.

CONCLUSION: Approximately 30 million people in North America meet the diagnostic criteria for IBS. However, data about the natural history of IBS is quite sparse and renewed efforts should be focused at developing appropriately designed trials of the epidemiology of IBS. (Am J Gastroenterol 2002;97:1910-1915. (C) 2002 by Am. Coll. of Gastroenterology)

The opinions and assertions contained herein are solely the views of the authors and should not be construed as official or as representing the views of the United States Government or the Department of Veteran Affairs.

\section{INTRODUCTION}

Irritable bowel syndrome (IBS) has been defined in the American Gastroenterological Association medical position statement as being "a combination of chronic or recurrent GI symptoms not explained by structural or biochemical abnormalities, which is attributed to the intestines and associated with symptoms of pain and disturbed defecation and/or symptoms of bloatedness and distention" (1). What makes IBS so challenging? The etiology is largely unknown, although different mechanisms for its symptoms have been proposed, including intestinal luminal irritants, psychological distress and psychiatric disease, postinfectious or postinflammatory phenomenon, and abnormal motor function $(2,3)$. More recently, abnormal visceral perception has been proposed as the mechanism behind the pain and abnormal defecation patterns (4). Because IBS lacks a "tangible lesion," IBS has been classified as a functional disorder and, as such, has the potential to be trivialized.

However, the relevance of IBS in clinical practice cannot be questioned. IBS accounts for $25-50 \%$ of referrals to gastroenterologists (5). National health surveys estimate that each year, there are 3 million physician visits, 2.2 million prescriptions, as well as 96,000 hospital discharges for IBS (6). One study from Olmsted County, MN, measured health care use in a random sample of the population and found that the charges incurred by people with IBS symptoms were, on average, $\$ 300$ higher than individuals without IBS (7). This figure, when extrapolated to the US population, would represent an excess of 8 billion dollars in medical costs among IBS patients. Therefore, knowledge of the epidemiology of IBS is clearly relevant to primary care providers, gastroenterologists, as well as policy makers.

Our purpose was to perform the first systematic review of published literature about the epidemiology of IBS in North America. Our specific study objectives were: 1) to determine the prevalence of IBS in North America, 2) to characterize the age of onset and gender distribution of IBS in North America, 3) to determine the incidence of IBS in North America, and 4) to characterize the natural history of IBS. Through this systematic review, limitations of current research may be identified and recommendations for future research may be suggested. 


\section{MATERIALS AND METHODS}

\section{Literature Search}

SEARCH STRATEGY FOR IDENTIFICATION OF STUDIES. A computer-assisted search of three online bibliographic databases was conducted to identify potentially relevant published papers. A search of the MEDLINE database from 1966 to present was performed using medical subject heading (MeSH) term "colonic diseases, functional" [mortality, ethnology, epidemiology] or "colonic diseases, functional" with exploded keywords including "incidence," "prevalence," "prognosis," and "natural history." Review of the EMBASE database from 1991 to 2000 was performed using the following terms: "[epidemiology]," "irritable colon," "incidence," "prevalence," "prognosis," and "history." The bibliographic database Current Contents/Science Edition was also searched between 1996 and 2001 using the keywords "irritable bowel syndrome," "IBS," "colonic diseases, functional," "functional colonic diseases," "irritable colon," "spastic colon," "incidence," "prevalence," "natural history," and "prognosis." Manual searches of reference lists from potentially relevant papers were also performed to identify any additional studies that may have been missed using the computer-assisted search strategy.

STUDY SELECTION CRITERIA. Two investigators (Y.S., G.R.L.) independently reviewed the titles and abstracts of all citations identified by the literature search. Potentially relevant studies were retrieved and the selection criteria applied. The selection criteria were: 1) studies of population-based samples of IBS patients derived within North America; 2) use of Manning, Rome I, or Rome II criteria to identify IBS patients; 3) population of adult patients (note that inclusion of pediatric patients within adult study population was allowed); 4) results reported on prevalence, incidence, or natural history of IBS; 5) published in full manuscript form; and 6) English language only.

CASE DEFINITION FOR DIAGNOSIS OF IBS. Only studies defining IBS using the Manning criteria and Rome criteria were included. The criteria for IBS proposed by Manning et al. in 1979 (Manning criteria) include the following six symptoms: 1) abdominal pain that is relieved with a bowel movement, 2) pain associated with looser stools, 3) pain associated with more frequent stools, 4) sensation of incomplete evacuation, 5) passage of mucus, and/or 6) abdominal distention (8). The number of symptoms required to meet the diagnosis was not stipulated. Using a threshold of two was associated with the highest sensitivity, whereas using a threshold of four was associated with the highest specificity.

Consensus criteria for the diagnosis of IBS have been established and subsequently modified by a panel of experts. The first set of such criteria was developed in conjunction with a meeting in Rome and, thus, these have become known as the Rome criteria. The original Rome criteria
("Rome I"), as published in 1989, required continuous or recurrent symptoms of abdominal pain that were 1) relieved with defecation, 2) associated with a change in frequency of stool, or 3) associated with a change in consistency of stool along with two or more symptom groups of disturbed defecation, which included: a) altered stool frequency, b) altered stool form, c) altered stool passage, d) passage of mucus, and e) usually accompanied by bloating or belief of abdominal distention (9). These criteria were subsequently revised and published in 1991 recommending that the pain be present for at least 3 months, that the symptoms of altered defecation occur at least $25 \%$ of the time, and that symptoms from at least three of the above five defecation disorder symptom groups be present ("modified Rome I") (10). These criteria were recently revised and called the Rome II criteria (11). Rome II criteria mandate $12 \mathrm{wk}$ of symptoms in the last year and require that two (rather than just one) of following features be associated with the pain: a) pain relieved by bowel movement, b) pain associated with harder or looser bowel movements, and/or c) pain associated with more or less frequent bowel movements. The defecation disorder symptom groups are now only supporting symptoms and not required for the diagnosis.

\section{Data Extraction and Data Analysis}

Eligible articles were reviewed in a duplicate, independent, unblinded manner by two investigators (Y.S., G.R.L.). Agreement between investigators was greater than 95\%, and disagreement in data extraction was resolved by consensus. For studies about the prevalence of IBS in North America, the prevalence, incidence, prevalence based on IBS subgroup, gender distribution, and the mean age of onset were extracted, if available. For prevalence studies, data about diagnostic definition of IBS (e.g., Manning criteria, ROME I criteria), sample size, and case ascertainment techniques to identify IBS patients (e.g., face-to-face interviews, mail surveys) was also extracted. For studies about the natural history of IBS, disease activity (e.g., prevalence of IBS over time and frequency of IBS flares within a specified period of time) and frequency of surgery or medication use was extracted.

Data extracted from original research articles about prevalence and gender distribution was converted into individual tables. The tables are presented in descriptive form. Because of significant variability in methods of reporting data, no attempt was made to pool the incidence or prevalence rates, gender distribution data, age at diagnosis data, or natural history of disease data.

\section{RESULTS}

\section{Characteristics of Selected Studies}

Search of the MEDLINE database yielded 232 articles. Review of the EMBASE database yielded 191 articles. Searching the Current Contents database yielded 157 articles with the above-described search. Reviews of the titles 
Table 1. Population-Based IBS Prevalence Studies in North America

\begin{tabular}{|c|c|c|c|c|c|c|}
\hline Study & Population & $\begin{array}{l}\text { Date of } \\
\text { Survey }\end{array}$ & Case Ascertainment & $\begin{array}{c}\text { Sample Size } \\
\text { (Response Rate) }\end{array}$ & Case Definition & $\begin{array}{c}\text { Prevalence } \\
\text { (per 100) }\end{array}$ \\
\hline Hahn et al. & NHIS & 1989 & Face-to-face interviews & 42,392 & $\begin{array}{l}\text { Manning } 2 \\
\text { Modified Rome I } \\
\text { Either criteria }\end{array}$ & $\begin{array}{l}8 \\
3 \\
8\end{array}$ \\
\hline Drossman et al. & U.S. Householder & 1990 & Phone interviews & $5430 / 8250(66 \%)$ & Modified Rome I & 9.4 \\
\hline Talley et al. & Olmsted County, MN & 1988 & Mailed questionnaires & $835 / 1021(82 \%)$ & $\begin{array}{l}\text { Manning } 2 \mathrm{p} \\
\text { Manning 3p } \\
\text { Manning 4p }\end{array}$ & $\begin{array}{r}17.0 \\
12.8 \\
8.7\end{array}$ \\
\hline Saito et al. & Olmsted County, MN & 1992 & Mailed questionnaires & $643 / 892(72 \%)$ & $\begin{array}{l}\text { Manning 2p } \\
\text { Manning 3p } \\
\text { Rome I } \\
\text { Modified Rome I }\end{array}$ & $\begin{array}{r}20.4 \\
15.7 \\
13.1 \\
8.5\end{array}$ \\
\hline
\end{tabular}

and abstracts, followed by review of the full manuscripts of potentially relevant articles, identified eight articles that met inclusion criteria (12-19). Only studies from the United States were found, as there were no published populationbased studies from Mexico or Canada.

\section{Prevalence Estimates by Definition}

The prevalence estimates for IBS varied between $3 \%$ and $20.4 \%$ (Table 1), with prevalence rates concentrated at approximately $10 \%$. In these studies, different definitions of IBS were used to quantify the prevalence of IBS. In the National Health Interview Survey (NHIS) performed in 1989, a total of 42,392 individuals were interviewed in a face-to-face manner to ascertain whether they experienced symptoms consistent with IBS (12). Using a threshold of two of six of the Manning symptoms, the US national prevalence was estimated to be $8 \%$ of the population. Using the modified Rome criteria, the prevalence was estimated to be $3 \%$. In the US Householder Survey published in 1993, the investigators surveyed 8250 households selected on the basis of a stratified probability random sample from a large consumer opinion mailing list (13). The modified Rome criteria were used to define cases of IBS. After excluding those reporting an organic disease, the prevalence for IBS was $9.4 \%$. Two population-based surveys have been conducted by mail in Olmsted County, MN. In the first study using a random sample of 1021 residents, 835 (82\%) eligible subjects responded (14). The definition of IBS was varied using thresholds of two, three, and four or more of the six Manning symptoms and the presence of frequent recurrent abdominal pain. Prevalence estimates after adjustment to the age and gender structure of the 1990 white population in the US were $17.0,12.8$, and 8.7 per 100 , respectively. In a follow-up study of this cohort, the prevalence estimates using Manning and Rome criteria were directly compared (15). The prevalence per 100 was 20.4 using a threshold of two Manning symptoms, 15.7 using three symptoms, 8.6 using four symptoms, 13.1 using the original Rome I criteria, and 8.5 using the modified Rome criteria.

\section{Gender Differences in Prevalence}

Prevalence estimates for each gender was reported in three studies (Table 2). The US Householders study found a higher prevalence in women; the prevalence among the women in their study was $14.5 \%$, versus $7.7 \%$ in men. In the NHIS, the ratio of female to male individuals with IBS was also approximately $2: 1$, although no specific estimates were given. However, in Olmsted County, the gender-specific

Table 2. Prevalence of IBS by Gender

\begin{tabular}{|c|c|c|}
\hline Study & Case Definition & Prevalence $(95 \% \mathrm{CI})$ \\
\hline Drossman et al. (US Householder) & Modified Rome I & $\begin{array}{l}\mathrm{F}: 14.5 \% * \\
\mathrm{M}: 7.7 \% *\end{array}$ \\
\hline \multirow[t]{2}{*}{ Talley et al. (Olmsted County, MN 1988) } & Manning 2p & $\begin{array}{l}\text { F: } 18.2(14.4-21.9) \\
\text { M: } 15.8(12.2-19.4)\end{array}$ \\
\hline & Manning 3p & $\begin{array}{l}\text { F: } 13.6(10.2-16.9) \\
\text { M: } 12.1(8.9-15.3)\end{array}$ \\
\hline \multirow[t]{5}{*}{ Saito et al. (Olmsted County, MN 1992) } & Manning 2p & $\begin{array}{l}\text { F: } 22.4(17.1-27.7) \\
\text { M: } 18.4(13.1-23.8)\end{array}$ \\
\hline & Manning 3p & $\begin{array}{l}\text { F: } 17.7(23.9-22.5) \\
\text { M: } 13.5(8.8-18.3)\end{array}$ \\
\hline & Manning 4p & $\begin{array}{l}\text { F: } 10.2(6.5-13.9) \\
\text { M: } 6.8(3.1-10.5)\end{array}$ \\
\hline & Rome I & $\begin{array}{l}\text { F: } 12.7(8.6-16.7) \\
\text { M: } 13.3(8.6-18.1)\end{array}$ \\
\hline & Modified Rome I & $\begin{array}{l}\text { F: } 8.4(5.2-11.7) \\
\text { M: } 8.4(4.3-12.4)\end{array}$ \\
\hline
\end{tabular}




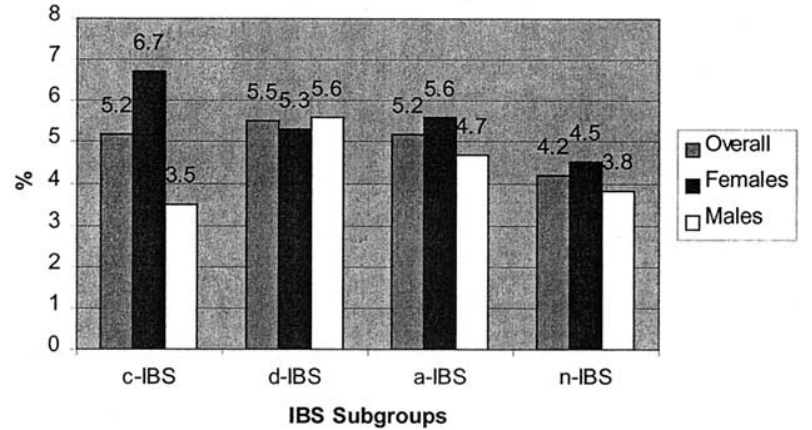

Figure 1. Age-adjusted prevalence of IBS (per 100 subjects) by symptom subgroups in Olmsted County, MN, 1988-1993. a-ibs = both constipation and diarrhea; c-ibs = constipation-predominant; $\mathrm{d}$-ibs = diarrhea-predominant; $\mathrm{n}$-ibs $=$ neither constipation nor diarrhea.

prevalence rates have been much more similar between men and women. In women, the prevalence was $18.2 \%$ as compared to $15.8 \%$ in males using the Manning 2 criteria, but the rates were very similar for women and men, $12.7 \%$ and $13.3 \%$, using the original Rome criteria.

\section{Age and Prevalence}

Each of the studies demonstrated slight changes in IBS prevalence by age. Younger persons had the highest prevalence, whereas prevalence was lower in the middle-aged population. In the US Householders study, the incidence was $13.5 \%$ in the group aged $<45 \mathrm{yr}$ compared with $9.4 \%$ in the group aged $>45 \mathrm{yr}$. Similar trends were seen in the Olmsted County population. However, in a separate study, a survey was mailed to a cohort of elderly Olmsted County residents and the prevalence (using three or more Manning criteria as the definition) increased slightly with age, from approximately $8 \%$ in the group aged 65-74 yr to more than $12 \%$ in the group aged $>85 \mathrm{yr}$ (16). Overall, these studies about the prevalence of IBS demonstrate minimal differences in IBS prevalence based on age. The prevalence of IBS was approximately $10 \%$, regardless of the age group.

\section{IBS Subgroups}

Only one study (17) has looked at the prevalence of IBS by symptom subgroups (Fig. 1). In a report that used data from a number of surveys of random samples of Olmsted County residents, the people who met the Manning criteria for IBS were further subdivided based on their bowel symptoms. The overall prevalence of constipation-predominant IBS was $5.2 \%$ and for diarrhea-predominant IBS it was 5.5\%. The prevalence of having both diarrhea and constipationtype symptoms was $5.2 \%$, whereas $4.2 \%$ of participants did not meet criteria for either constipation or diarrhea. The age-adjusted, gender-specific prevalence rates were similar for men and women except in the constipation-predominant IBS group, in which the prevalence was significantly higher in women $(6.7 \%$ vs $3.5 \%)$. In the Epidemiology of Constipation study (EPOC), a total of 10,018 persons selected from across the US were interviewed by telephone (18). The prevalence for the IBS in the constipation subgroup was $2.1 \%$; the prevalence for those reporting a combination of IBS and rectal outlet obstruction symptoms was $3.5 \%$.

\section{Incidence of IBS}

No true population-based North American IBS incidence studies were found in this literature search. A single study used repeated surveys over time to estimate incidence (19). In Olmsted County, a cohort was surveyed on two occasions $1 \mathrm{yr}$ apart, using the Manning criteria, and 9\% of subjects free of IBS at baseline reported IBS in follow-up. This was calculated to be an onset rate of 67 per 1000 person-years. However, this study did not determine whether study patients had a past medical history of IBS. These surveys, which report the development of IBS symptoms over the course of $1 \mathrm{yr}$, determine only whether the study patients had active IBS at the onset of the study. Thus, these respondents may not have been true "incident" cases with first-time onset of symptoms.

\section{Natural History}

The same study, which used repeated surveys over a 1-yr interval, evaluated natural history of IBS and met inclusion criteria for this systematic review. In this longitudinal study done in Olmsted County, 38\% of subjects who met the definition of IBS (using two or more Manning criteria) did not meet the criteria at 1-yr follow-up. Over 1 year, the prevalence using the Manning criteria changed only from $17 \%$ to $18.1 \%$. Using the modified Rome criteria, the prevalence changed from $7.2 \%$ to $8.0 \%$. Thus, there is a relative numerical balance in the number of persons who begin to meet and fail to meet the definition of IBS over time. No other study of North American IBS patients provides data about other aspects of natural history. Thus, no study in this systematic review provides data about disease activity of IBS or about frequency of surgery or medication use.

\section{DISCUSSION}

Our systematic review of the literature has shown that the prevalence estimates for IBS in the United States have ranged from $3 \%$ to $20 \%$; however, this wide range of prevalence estimates seems to be the result of changes in the definition of IBS used rather than true differences in IBS prevalence. No one definition can be considered a gold standard for the diagnosis, although the hope is that Rome II will become such. Unfortunately, the studies in this review were performed before the development of the Rome II criteria. Nevertheless, most estimates indicate that the prevalence of IBS is approximately $10-15 \%$, and this estimate is consistent with multiple non-US IBS studies summarized in previous review articles (20). An IBS prevalence of $10-15 \%$ estimates that as many as $30-45$ million North American adults have symptoms of IBS during any given year. Yet, this systematic review is marked by the sparse data about the natural history of IBS in North America, 
including the incidence of IBS, disease activity or frequency of surgery, and medication use. Given the relative lack of data, this systematic review reinforces the need for appropriately designed trials to clarify the epidemiology of IBS.

This review has shown fairly consistent results in the prevalence estimates by age, gender, and symptom subgroup. The prevalence decreases slightly with age in most trials, and the prevalence is similar for constipation-predominant IBS and diarrhea-predominant IBS. Unlike clinicbased studies (21), population-based studies do not demonstrate the $4: 1$ female to male predominance. Rather, in the community, this ratio ranges from 1:1 to $2: 1$. The underlying reasons behind these gender differences are unclear. They could, again, be related to criteria used for diagnosis (15); or they could be related to sociocultural differences, differences in health-care seeking behavior between men and women, or true biological differences in the natural history of IBS between men and women. Future epidemiological research should seek to elicit possible reasons underlying these findings.

Appropriately designed trials about the incidence of IBS in North America are lacking. One population-based study has been reported by one of the authors (G.L.) but presented only in abstract form (22). The incidence of a clinical diagnosis of IBS was determined in Olmsted County by identifying persons with a diagnosis of IBS in the medical record, and reviewing their medical records to be sure that the person had not received a diagnosis of IBS in the preceding decade. The incidence was found to be 200 per 100,000 person-years, although this estimate is likely to be lower than the true incidence of IBS for reasons discussed below.

Optimal natural history studies would provide data about the disease activity of IBS, frequency of surgery and medication use, or even the frequency that IBS symptoms cause these individuals to miss work. Although no studies that met our prespecified criteria provided this information, other sources do provide some estimates about the natural history of IBS. IBS is an episodic disorder, marked by periods of severe disease activity followed by periods of minimal symptoms. A single study has examined the natural history of IBS symptoms during a period of severe IBS disease activity (23). In this study, 122 IBS patients in the United Kingdom who actively sought care for symptomatic IBS were followed for $12 \mathrm{wk}$. The study patients reported approximately 12 distinct "episodes" of IBS symptoms over the 12-wk period. Maximum duration of each IBS episode was 5 days, although the majority of study patients reported IBS symptoms on more than $50 \%$ of days. Thus, in this trial in the United Kingdom, patients alternate between several days with symptoms and several days without symptoms. The results from the single IBS natural history trial (including those in this systematic review) demonstrated that up to $38 \%$ of IBS patients did not meet diagnostic criteria for IBS, and reported prolonged symptom-free periods after $1 \mathrm{yr}$ of follow-up, suggesting that IBS patients may develop a "re- mission" after a series of symptomatic episodes (19). Longitudinal studies have also been done outside the US, and one study in particular bears reporting (24). A Swedish team surveyed individuals on two occasions $1 \mathrm{yr}$ apart, and found symptom onset and disappearance rates similar to those in the previous study in Olmsted County, MN. This study went a step further by showing that the symptoms changed over time. The $40 \%$ of individuals who were said to have "disappearance" of IBS symptoms actually had other GI symptoms during follow-up, and the $10 \%$ who had "onset" of IBS often had other symptoms at baseline. Thus, the coming and going of symptoms is not an all-or-none phenomenon; rather, there is a great deal of symptom change.

Data about the frequency of surgery or medication use or even the frequency of missed work because of IBS symptoms are also part of the natural history of IBS, although no population-based trials of North American patients report these data in full manuscript form. Nevertheless, prescription databases suggest that a low estimate of the cost of pharmaceutical use by IBS patients exceeds 80 million dollars annually, with total costs of health care exceeding 1 billion dollars annually (25). The US Householder Survey (13) estimated that IBS patients miss 13 days of work annually because of illness, compared with control patients who miss only 5 days of work annually because of illness. If the additional work loss resulting from illness is attributable to IBS, then the indirect costs from this absenteeism may exceed 19 billion annually (25). Finally, additional indirect data suggests that IBS patients are more likely to undergo abdominal surgical procedures. Specifically, one population-based trial in the United Kingdom found that cholecystectomy was more common in IBS patients compared with controls ( $4.6 \%$ vs $2.4 \%$, respectively; $p<0.01$; $\mathrm{OR}=1.9,95 \% \mathrm{CI}=1.2-3.2)(26)$. IBS patients are more likely to undergo hysterectomy (27) and appendectomy (28) compared with controls. Finally, based on recent abstract data, the lifetime prevalence of abdominal surgery in IBS patients may be two times higher when compared with normal controls (59\% vs 32\%, respectively) (29).

Given the paucity of literature about the incidence and natural history of IBS, renewed efforts should focus on the development of well-designed trials about the epidemiology of this disease. These trials will need to overcome several potential pitfalls. First, in the prevalence studies, only 50\% of people with IBS symptoms report having ever seen a physician for these symptoms; thus, medical records cannot identify everyone with IBS. Symptoms may come and go; thus, symptom inventories must be taken repeatedly over time. Recent studies have identified that IBS symptoms are common in both middle school and high school aged individuals (30). Thus, a true IBS incidence study would require data collection to begin almost at birth. Therefore, optimal trials about the epidemiology of IBS would meet the following criteria for a natural history study: 1) populationbased samples of patients followed from the time of diagnosis, 2) appropriate and objective criteria for the initial 
diagnosis of disease, and 3) sufficiently long and complete follow-up of the cohort of patients using objective definitions of disease activity and other outcomes (31). Appropriate trial outcomes may include assessment of disease activity, medication use, health care resource use, frequency of surgery, and frequency of absenteeism from work because of IBS symptoms.

Thus, there is clearly need for a prospective cohort study to determine the exact incidence and natural history of IBS. Ideally, this study should be community based, with at least an annual survey collecting information regarding the presence of symptoms and their severity and frequency, as well as collecting information regarding morbidity, mortality, and health care use. Otherwise, quantifying the natural history of symptoms in a prospective manner will be impossible. These studies and others suggest that the health care burden of IBS is great in the US population. Further investigation of the true health and financial burden of IBS should be performed.

Reprint requests and correspondence: G. Richard Locke, M.D., Mayo Clinic, 200 First Street, SW, Rochester, MN 55905.

Received Dec. 26, 2001; accepted Jan. 7, 2002.

\section{REFERENCES}

1. American Gastroenterological Assocation. American Gastroenterological Association medical position statement: Irritable bowel syndrome. Gastroenterology 1997;112:2118-9.

2. Camilleri M, Prather CM. The irritable bowel syndrome: Mechanisms and a practical approach to management. Ann Intern Med 1992;116:1001-8.

3. Lind CD. Motility disorders in the irritable bowel syndrome. Gastroenterol Clin North Am 1991;20:279-95.

4. Naliboff BD, Munakata J, Fullerton S, et al. Evidence for two distinct perceptual alterations in irritable bowel syndrome. Gut 1997;41:505-12.

5. Mitchell CM, Drossman DA. Survey of the AGA membership relating to patients with functional gastrointestinal disorders. Gastroenterology 1987;92:1282-4.

6. Sandler RS. Epidemiology of irritable bowel syndrome in the United States. Gastroenterology 1990;99:409-15.

7. Talley NJ, Gabriel SE, Harmsen WS, et al. Medical costs in community subjects with irritable bowel syndrome. Gastroenterology 1995; 109:1736-41.

8. Manning AP, Thompson WG, Heaton KW, et al. Towards positive diagnosis of the irritable bowel. Br Med J 1978;2: $653-4$.

9. Thompson WG, Dotevall G, Drossman DA, et al. Irritable bowel syndrome: Guidelines for diagnosis. Gastroenterol Int 1989;2:92-5.

10. Drossman DA, Thompson WG, Talley NJ, et al. Identification of sub-groups of functional gastrointestinal disorders. Gastroenterol Int 1990;3:159-72.

11. Thompson WG, Longstreth GF, Drossman DA, et al. Functional bowel disorders and functional abdominal pain. Gut 1999;45(suppl II):II43-7.
12. Hahn BA, Saunders WB, Maier WC. Differences between individuals with self-reported irritable bowel syndrome (IBS) and IBS-like symptoms. Dig Dis Sci 1997;42:2585-90.

13. Drossman DA, Zhiming L, Andruzzi E, et al. U.S. householder survey of functional gastrointestinal disorders. Dig Dis Sci 1993;38:1569-80.

14. Talley NJ, Zinsmeister AR, Van Dyke C, et al. Epidemiology of colonic symptoms and the irritable bowel syndrome. Gastroenterology 1991;101:927-34.

15. Saito YA, Locke GR, Talley NJ, et al. A comparison of the Rome and Manning criteria for case identification in epidemiological investigations of irritable bowel syndrome. Am J Gastroenterol 2000;95:2816-24.

16. Talley NJ, O Keefe EA, Zinsmeister AR. Prevalence of gastrointestinal symptoms in the elderly: A population-based study. Gastroenterology 1992;102:895-901.

17. Talley NJ, Zinsmeister AR, Melton LJ. Irritable bowel syndrome in a community: Symptom subgroups, risk factors, and healthcare utilization. Am J Epidemiol 1995;142:76-83.

18. Stewart WF, Liberman JN, Sandler RS, et al. Epidemiology of Constipation (EPOC) Study in the United States: Relation of clinical subtypes to sociodemographic features. Am J Gastroenterol 1999;94:3530-40.

19. Talley NJ, Weaver AL, Zinsmeister AR, et al. Onset and disappearance of gastrointestinal symptoms and functional gastrointestinal disorders. Am J Epidemiol 1992;136:165-77.

20. Drossman DA, Whitehead WE, Camilleri M. Irritable bowel syndrome: A technical review for practice guideline development. Gastroenterology 1997;112:2120-37.

21. Camilleri M. Management of irritable bowel syndrome. Gastroenterology 2001;120:652-68.

22. Locke GR, Yawn BP, Wollan PC, et al. The incidence of clinically diagnosed IBS in the community. Gastroenterology 1999;116:A76 (abstract).

23. Hahn B, Watson M, Yan S, et al. Irritable bowel syndrome symptom patterns: Frequency, duration, and severity. Dig Dis Sci 1998;43:2715-8.

24. Agreus L, Svardsudd K, Nyren O, et al. Irritable bowel syndrome, and dyspepsia in the general population. Overlap and lack of stability over time. Gastroenterology 1995;109:67180.

25. American Gastroenterological Association. The burden of gastrointestinal illness. Bethesda, MD: AGA Press, 2001.

26. Kennedy TM, Jones RH. Epidemiology of cholecystectomy and irritable bowel syndrome in a UK population. Br J Surg 2000;87:1658-63.

27. Longstreth GF, Preskill DB, Youkeles L. IBS in women having diagnostic laparoscopy or hysterectomy: Relation to gynecologic features and outcome. Dig Dis Sci 1990;35:128590.

28. Burns DG. The risk of abdominal surgery in IBS. S Afr Med J 1986;70:91.

29. Olden K, Chey W, Boyle J, et al. Health-related quality of life in IBS: A community based study. Gastroenterology 2001; 120:A634 (abstract).

30. Hyams JS, Burke G, Davis PM, et al. Abdominal pain and irritable bowel syndrome in adolescents: A community-based study. J Pediatr 1996;129:220.

31. Laupacis A, Wells G, Richardson WS, et al. Users' guides to the medical literature. How to use an article about prognosis. JAMA 1994;272:234-7. 